\title{
A Review of Antibiotic Residue Determination in Aquatic Products
}

\author{
Yuanmin $\mathrm{Mo}^{1,3, a}$, Liangliang Huang ${ }^{1,2, b^{*},}$, Mingxing Deng ${ }^{1,3}$, Jian Huang ${ }^{2,3}$ \\ and Saeed $\operatorname{Rad}^{1}$ \\ ${ }^{1}$ College of Environmental Science and Engineering, Guilin University of Technology, Guilin \\ 541000, China \\ ${ }^{2}$ Guangxi Key Laboratory of Environmental Pollution Control Theory and Technology, Guilin \\ University of Technology, Guilin 541000, China \\ ${ }^{3}$ Collaborative Innovation Center for Water Pollution Control and Water Safety in Karst Area, Guilin \\ University of Technology, Guilin 541000, China \\ ayuanminmo@163.com, ${ }^{\mathrm{b} l l h u a n g @ g l u t . e d u . c n ~}$ \\ Corresponding author: Liangliang Huang
}

Keywords: aquatic products, antibiotics, determination.

\begin{abstract}
In recent years, with the development of fisheries, the aquaculture industry has developed rapidly and has become an important part of our country economy. At the same time more and more aquatic products is being used by people while the abuse of antibiotics is growing uncontrolled. This paper summarizes the detection methods of various kinds of antibiotics in aquatic products, as well as the method of spiked recovery, detection limit, quantitative limit, and puts forward the corresponding countermeasures proposal of controlling the abuse of antibiotics.
\end{abstract}

\section{Introduction}

Veterinary drug residue analysis of meat and seafood products is an important part of national regulatory agency food safety programs to ensure that consumers are not exposed to potentially dangerous substances [1]. Toussaint et al. (2005) established a method for determination of quinolone antibiotic residues in pig kidney using liquid chromatography-tandem mass spectrometry [2]. Hammel et al. (2008) developed a multi-screening approach for monitoring potential chemical contaminants in honey by liquid chromatography - electrospray ionization tandem mass spectrometry [3]. Draisci et al. (2001) created a new sensitive electrochemical enzyme-linked immunosorbent assay (ELISA) for detection of two macrolides (erythromycin and tylosin) in bovine muscle [4]. Okerman et al. (2003) analyzed antibiotic residue of bovine and porcine kidney tissue by a solid-phase fluorescence immunoassay. However, pharmaceuticals identified as emerging contaminants are used in large quantities in human and veterinary medicine for treatment of different diseases [5]. Among pharmaceuticals, antibiotics in the aquatic environment is a big challenge as prolonged exposure to low does may promote antibiotic resistance [6]. The accelerated growth of aquaculture has resulted in a series of harmful effects to human health. The widespread and unrestricted use of antibiotics in this industry, to prevent bacterial infections [7], has leaded some excess amount of unutilized antibiotics to remain in the environment. The use of antibiotics and drugs in fish farms has prompted an investigation into the elimination of these residues from fish muscle [8]. Won et al. (2011) analyzed both domestic and imported marine products in Korea for 14 sulfonamide antimicrobials by UPLC-MS / MS and found out that the samples include flatfish, jacopever, sea bream, common eel, blue crab, shrimp and abalone [9]. Swapna et al. (2012) detected the antibiotic residues of farmed shrimps from the southern states of India. The results were showing that streptomycin, tetracycline and $\beta$ - lactam could not be detected in any of the samples and sulfonamides and erythromycin were detected in farmed shrimps at a level $<100 \mathrm{ppb}$ [10]. Such findings are not limited to abroad, there are many studies on detecting antibiotics in China too.

The case in China is much more sensitive in compare to other regions as China is not just a big consumer but a large producer of antibiotics too. With the decline of fishery resources, aquaculture 
has gradually become a major food industry to partially solve the "food shortage" and to provide food safety [11]. In the past three decades, aquaculture industry has developed rapidly, and the output value of aquatic products has increased from $1.30 \%$ in 1952 to $11.19 \%$ in 2003 . The aquatic productions ranks as first in the world's aquaculture production and has reached 35.97 million tons in 2006 . These statistics are based on $68 \%$ of total fisheries production and $70 \%$ of the world's total aquaculture production [12]. Antibiotics are widely used in aquaculture. The positive effects of antibiotics are mainly reflected in the prevention and control of aquatic animal diseases, promoting growth, saving nutrition and other aspects. Especially that it can efficiently control the occurrence of many aquatic diseases and promote the development of aquaculture. This paper mainly introduces the rapid detection of antibiotics in aquatic products in China.

\section{Aquatic Products}

\subsection{Fish}

Lin et al. (2005) established the determination of sulfonamides veterinary drug residues ineel and roasted eel, including sulfamerazine, sulfadimidine, sulfamethoxypyridazine, sulfamet-hoxazole, sulfamonomethoxine, sulfadimethoxine, sulfaquinoxaline by high performance.

Liquidchromatography [13].The sample was extracted with dichloromethane and the recoveries were in the range of $80 \% \sim 93 \%$. The LOD was $0.02 \mathrm{mg} / \mathrm{kg}$. And the LOQ was $0.05 \mathrm{mg} / \mathrm{kg}$.

Ji et al. (2005) established a method to determine residues of tetracycline, chlortetracycline, oxytetracycline, doxycycline, demeclocycline, methacycline and minocycline in aquatic products [14]. The samples were extracted by EDTA-McIlvaine buffer solution and were purified by HLB solid-phase extraction column. The Inertsil C8-3 reversed-phase column was used. The mobile phase was methanol-acetonitrile $+0.01 \mathrm{~mol} / \mathrm{L}$ trifluoroacetic acid. The detection limit of this method was $1.5 \sim 5.0 \mu \mathrm{g} / \mathrm{kg}$. The recoveries were $75.2 \% \sim 100.6 \%$ and the relative standard deviations were $2.19 \%$ $\sim 10.56 \%$.

Yang et al. (2005) established a method for the determination of florfenicol residues in eel by HPLC. The samples were extracted by ethyl acetate and cleaned up by C18 solid-phase extraction cartridge. The detection limit of this method was $9.1 \mu \mathrm{g} / \mathrm{kg}$, the limit of quantification was $22.3 \mu \mathrm{g} / \mathrm{kg}$ [15]. The average recovery was more than $90 \%$ and the relative standard deviation of $4.24 \%$ to $7.36 \%$.

A high-performance liquid chromatography tamden mass spectrometric method was used to determine the residues of quinocetones, olaquindox and 5 kinds of macrolides antibiotics in crucian carp and turtle [16]. The mixture was extracted with the mix solution of ethyl- acetate and acetonitrile and cleaned up by $\mathrm{N}$-hexane. The mobile phase is acetonitrile $-10 \mathrm{mmol} / \mathrm{L}$ ammonium formate solution. The recoveries of this method were about $67.52 \% \sim 108.89 \%$ and the relative standard deviations were $4.2 \% \sim 14 \%$. The LOD was $1 \mu \mathrm{g} / \mathrm{kg}$ and LOQ was $2 \mu \mathrm{g} / \mathrm{kg}$.

A method for simultaneous determination of four kinds of antibiotics in aquatic products by SPE high performance liquid chromatographic method was established. The method is based on ZORBAXSPE C18 Cart as solid phase extraction column and SUPELCO Discovery C18 as the chromatographic column. The mobile phase was $0.01 \mathrm{~mol} / \mathrm{L} \mathrm{NaH}_{2} \mathrm{PO}_{4}$ solution and a mixture solution of $0.001 \mathrm{~mol} / \mathrm{L}$ EDTA and Methanol [17]. The recoveries were above $90 \%$ and the relative standard deviation was less than $2 \%$.

Zhang et al. (2013) applied a method for rapid determination of nitrofuran metabolites in aquatic products using colloidal gold immune chromatography technology. The detection limits of AOZ, SEM, AMOZ and AHD were 1.0, 1.0 and $2.0 \mu \mathrm{g} / \mathrm{kg}$ respectively [18].

A method for the determination of olaquindox in aquaticindustry by high performance liquid chromatography was used by Liu et.al.(2017). The sample was extracted by $5 \%$ methanol solution and cleaned up by HLB solid phase extraction column and N-hexane. The mobile phase was $15 \%$ methanol solution. The recoveries were about $83.2 \% \sim 94.4 \%$ and the relative standard deviations were about $2.2 \% \sim 7.7 \%$ The LOD and LOQ were $0.02 \mathrm{mg} / \mathrm{kg}$ and $0.05 \mathrm{mg} / \mathrm{kg}$ respectively.

\subsection{Shrimp and Crab}

Wang et.al. (2015) established a method for determination of antibiotic residues of Leander modestus Heller and Macrobrachium Nipponense in Chaohu Lake by high performance liquid 
chromatography - tandem mass spectrometry. The sample were purified with N-hexane and extracted by a mix solution of acetonitrile and dichloromethane (95:5). Five kinds of antibiotics were quantified as sulfadiazine, sulfachoropyridazine, lomefloxacin, enrofloxacin and ofloxacin. The average concentration was about $0.21 \sim 1.94 \mu \mathrm{g} / \mathrm{kg}$. The recoveries of $25 \mathrm{ug} / \mathrm{kg} \mathrm{t}$ and $250 \mathrm{ug} / \mathrm{kg}$ were $58 \% \sim$ $104 \%$ and $58 \% \sim 102 \%$ respectively and the limit of quantification was $0.14 \sim 11.64 \mathrm{ug} / \mathrm{kg}$.

Zhang et.al. (2012) applied a method for determination of quinolone antibiotic residues ofshrimps and crabs. The samples were extracted by Mellvaine buffer solution and purified byHLB solid-phase extraction column. The samples were then tested by ultra performance liqu-id chromatography / mass spectrometry. The concentration was about $1.0 \sim 53.6 \mathrm{ug} / \mathrm{kg}$.

Another method for the determination of tetracycline antibiotics residues in nine kinds of lobster shrimps was used by Su et al. (2016). The mobile phase were $0.05 \%$ formic acid acetonitrile and $0.05 \%$ formic acid water. The samples were extracted by 5\% trichloroacetic acid-phosphate solution and cleaned up by HLB solid phase extraction column. The recoveries were in the range of $66.1 \%$ $107.9 \%$, and the RSD were between $0.7 \%$ and $9.7 \%$ [22].

Liu et al. (2010) established a high performance liquid chromatography tandem/mass spectrometry method for the detection of antibiotic residues in Penaeus vannamei Boone. The samples were extracted by a mixed solution of ethyl acetate - acetonitrile and puried by N-hexane. The mobile phase was the acetonitrile $-10 \mathrm{mmol} / \mathrm{L}$ ammonium format solution. The recoveries were $67.5 \% \sim 108.9 \%$ at the three spiked levels. The relative standard deviation was about $4.2 \% \sim 14 \%$. The LOD was 1 $\mathrm{ug} / \mathrm{kg}$, and the LOQ was $2 \mathrm{ug} / \mathrm{kg}$ [16].

Lin et al. (2009) detected the tetracycline antibiotic residues of aquatic products in Guangzhou City by high performance liquid chromatography. The residual concentration was $0.37 \sim 11.5 \mathrm{mg} /$ $\mathrm{kg}$, and the residual rate of shrimp and crab was the lowest, which was $48.68 \%$.

\section{Summary}

Not just fish and shrimp, there are many types of aquatic products, such as shellfish, algae and etc. Most of antibiotic studies have focused on fish, shrimp, crabs and shellfish, however other aquatic products which are being consumed also must be taken into consideration. Aquaculture has an important role for people in economy and production point of view. According to the United Nations Food and Agriculture Organization (FAO), it has been estimated that the excessive exploitation of offshore fisheries led to a decline in catches and has also been forecasted that half of the world's seafood will come from aquaculture until the year of 2020 [24].

A variety of antibiotics are often thrown into aquaculture water by farmers, because it treats most of the diseases caused by bacterial infections such as bacteria, rickettsia, mycoplasma, chlamydia, spirochetes and other microbial infections. Since 1940 which penicillin G was first used to treat bacterial infections, antibiotics have played an increasingly important role in the prevention and treatment of diseases caused by bacteria and other microorganism. However, the use of antibiotics has also brought a series of problems. In general, only a small part of antibiotics remain inside the animal body after entering into it and the rest are left in the sediments of aquaculture. Many scholars have reported that antibiotics have the accumulation effect and And a certain accumulated amount can produce toxic effects on animals [12]. Therefore when aquatic products (which contain all types of antibiotics) are used by human, it might cause accumulation of antibiotics and consequently lead to public health issues. The potential risks of antibiotics can not be underestimated. Arican et al. (2009) have been done a research on reducing chlortetracycline residues in beef manure with composting [25]. These phenomena can be addressed from the following aspects: (1) The government should strengthen the supervision of fish drugs usage, increase the punishment of illegal use of antibiotics and establish more simultaneous testing method of different types of antibiotics. The government also should vigorously implement the GMP certification system of veterinary medicine, strengthen the source management of antibiotic production and improve the overall level of veterinary drug quality [26]. (2) Farmers education on applying antibiotics must be considered in order to follow the rules specially on reduction of using veterinary drugs during withdrawal period. As per literatures states provide withdrawal period for some veterinary drugs, especially drug additives, but some farmers 
will use the drug containing additives without considering the provisions of withdrawal period [26]. (3) To explore and develop more antibiotic substitutes, such as micro-ecological preparations and etc are recommended.

\section{Acknowledgements}

This work was financially supported by the National Natural Science Foundation of China (51509042), Natural Science Foundation of Guangxi (2016GXNSFAA380104) and Dr. Scientific Research Foundation and High - level Innovation Team and Scholars Program of Guangxi Higher Education.

\section{References}

[1]. Lohne J.J., Andersen W.C., Clark S.B. , et al. Laser diode thermal desorption mass spectrometry for the analysis of quinolone antibiotic residues in aquacultured seafood. Rapid Communications in Mass Spectrometry. Vol. 26 (2012) No. 24, p.2854-2864.

[2]. Toussaint B. , Chedin M. , Bordin G. , et al. Determination of (fluoro)quinolone antibiotic residues in pig kidney using liquid chromatography-tandem mass spectrometry. I. Laboratoryvalidated method. Journal of Chromatography A. Vol. 1088 (2005) No. 1-2, p. 32-39.

[3]. Hammel Y.A., Mohamed R. , Gremaud E. , et al. Multi-screening approach to monitor and quantify 42 antibiotic residues in honey by liquid chromatography-tandem mass spectrometry. Journal of Chromatography A. Vol. 1177 (2008) No. 1, p. 58-76.

[4]. Draisci R. , Delli Q.F., Achene L., et al. A new electrochemical enzyme-linked immunosorbent assay for the screening of macrolide antibiotic residues in bovine meat. Analyst. Vol. 162 (2001) No. 11, p. 1942-1946.

[5]. Okerman L. , De W.K. , Van H.J., et al. Simultaneous determination of different antibiotic residues in bovine and in porcine kidneys by solid-phase fluorescence immunoassay. Journal of Aoac International. Vol. 86 (2003) No. 2, p. 236-240.

[6]. Hernandez F. , Sancho J.V. , Ibanez M. , et al. Antibiotic residue determination in environmental waters by LC-MS. Trend in Analytical Chemistry. Vol. 26 (2007) No. 6, p. 466-484.

[7]. Samanidou V.F. , Evaggelopoulou E.N. . Analytical strategies to determine antibiotic residues in fish. Journal of Separation Science. Vol. 30 (2007) No. 30, p. 2549-2569.

[8]. McCracken A. , Fidgeon S. , O'Brien J.J. , et al. An investigation of antibiotic and drug residues in fish. Journal of Applied Bacteriology. Vol. 40 (1976) No. 1, p. 61-66.

[9]. Won S.Y., Lee C.H., Chang H.S. , et al. Monitoring of 14 sulfonamide antibiotic residues in marine products using HPLC-PDA and LC-MS/MS. Food Control. Vol. 22 (2011) No. 22, p. 1101-1107.

[10]. Swapna K.M. , Rajesh R. , Lakshmanan P.T. . Incidence of antibiotic residues in farmed shrimps from the southern states of India Indian. Journal of Geo-Marine Sciences. Vol. 41 (2012) No. 4, p. 344-347.

[11]. Zhang Xiaoshuang, Li Nan, Cai Wengui, et al. Cause and advise on aquaculture water pollution in China. Chinese Fisheries Economics. (2007) No. 5, p. 30-33.

[12]. Chen Qinghua. Risks and control of antibiotic use in aquaculture. Fisheries Science and Technology Information. Vol. 36 (2009) No. 2, p. 67-72.

[13]. Lin Haidan, Xie Shouxin, Wu Yingxuan. Determination of sulfonamides veterinary drugs residues in eels and roasted eels by SPE-HPLC. Food Science. Vol. 26 (2005) No. 1, P. 176-179.

[14]. Ji Caini, Yue Zhenfeng, Xie Liqi, et al. Study on simultaneous detection of 7 tetracyclines residues in aquatic products by high performance liquid chromatography. Chinese Journal of Veterinary Science and Technology. Vol. 35 (2005) No. 10, p.820-826.

[15]. Yang Fang, Yu Kongjie, Huang Jiansheng. Determination of florfenicol residue in eel muscle by HPLC. Chinese Journal of Analysis Laboratory. Vol. 24 (2005) No. 2, p. 44-46.

[16]. Liu Yongtao, Liu Zhenhong, Ding Yunmin, et al. Simultaneous determination of residue of quinocetone olaquindox and five kinds of macrolide antibiotics in fishery products by high 
performance liquid chromatography tandem mass- spectrometry. Chinese Journal of Analysis Laboratory. Vol. 29 (2010) No.8, p. 44-47.

[17]. Luo Xiaoyan, Lin Yuna, Liu Lizhi. Study on the rapid determination of 4 kinds of antibiotics residues in aquaculture by SPE-HPLC. Modern Preventive Medicine. Vol. 32 (2005) No.3, p. 198-220.

[18]. Zhang Min, Sang Liya, Wang Zhenguo, et al. Development and application of colloidal gold rapid test strip for nitrofuran metabolites in aquatic products. Chinese Journal of Food Hygiene. Vol. 25 (2013) No. 1, p. 32-36.

[19]. Liu Wenxia, Zheng Jingjiao. Determination of olaquindox residue in aquatic water by HPLC. Science and Technology and Innovation. (2017) No.9, p. 137-138.

[20]. Wang Huazhang, Wang Chenchen, Tang Jun. Investigation of antibiotics residues in aquatic food in Chaohu Lake by HPLC-MS / MS. Journal of Anhui Agriculture. Vol. 43 (2015) No.22, p. 94-95, 105.

[21]. Zhang Qiuping, Wang Chunmin, Li Jian. Status of quinolones residues in animal food in Suzhou City. Occup and Health. Vol. 28 (2012) No. 18, p. 2257-2258.

[22]. Su Jing, Tang Lizhong, Chen Changyi, et al. Simultaneous determination of nine aminoglycosides and tetracyclines antibiotics residues in crayfish by high performance liquid chromatography-tandem mass spectrometry. Science and Technology of Food Industry. Vol. 37 (2016) No. 2, p. 60-67.

[23]. Lin Xiaohua, Lin Yingyue, He Jieyi, et al. Survey of hormmone and antibiotic residual in animal products in Guangzhou. China Tropical Medicine. Vol. 9 (2009) No. 10, p. 2081-2082.

[24]. Frankic A., Hershner C. . Sustainable aquaculture:developing the promise of aquaculture. Aquaculture International. Vol. 11 (2009) No. 6 , p. 517-530.

[25]. Arikan O.A. , Mulbry W. , Ric C. . Management of antibiotic residues from agricultural sources: use of composting to reduce chlortetracycline residues in beef manure from treatedanimals. Journal of Hazardous Materials. Vol. 164 (2009) No. 2-3, p. 483-489.

[26]. Chen Jingxiong, Yue Jianqun. Current situation and control of antibiotics usage in Aquaculture. Chinese Abstract of Animal Husbandry and Veterinary Medicine. Vol. 29 (2013) No. 5, p. 15-16. 